\section{Nettie Maria Stevens \\ (1861-1912)}

Simone Gilgenkrantz

$>$ Nettie Stevens fut une des premières femmes à apporter sa contribution aux recherches en génétique: elle découvrit la fonction des chromosomes sexuels durant la première décennie du XXe siècle. Mais son rôle resta méconnu et elle ne reçut pas la reconnaissance qu'elle aurait légitimement méritée, éclipsée par T.H. Morgan à qui fut accordé tout le crédit de la découverte de la génétique moderne. En France, où son nom est absent de la plupart des livres de biologie et de génétique, il nous a semblé légitime de lui rendre hommage par cette courte biographie. <

Tout ce que nous voulions savoir sur les chromosomes sexuels, nous sommes convaincus de l'avoir appris. En 1923, Théophile Painter découvrait le chromosome y dans les divisions méiotiques de spermatogonies [1] de testicules humains ${ }^{1}$. La garniture gonosomique, XX pour la femme et XY pour l'homme, était donc connue avant même qu'on sache le nombre exact de chromosomes humains, qui fut longtemps estimé de façon erronée à 48.

Auparavant, de nombreux chercheurs avaient étudié les chromosomes des plantes et des invertébrés. Les premières années du $X X^{e}$ siècle furent, pour l'hérédité, une période féconde. Hypothèses et théories venaient se mesurer aux descriptions faites sur le vif par les botanistes et les zoologistes. En Allemagne, Carl Correns (1864-1933) faisait connaître les travaux de Mendel qu'il venait

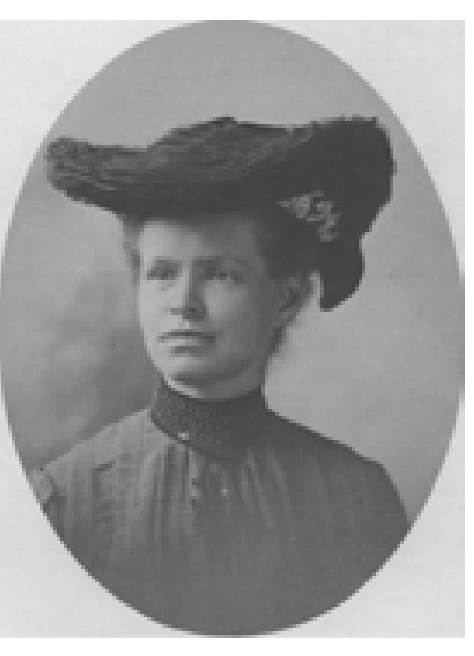

Nettie Maria Stevens, Photographie prise en 1904

(Courtesy of the Carnegie Institution of Whashington).
Médecine/Sciences,

9 , rue Basse,

54330 Clérey-sur-Brénon, France.

simone.gilgenkrantz@club-internet.fr rédité. Aux États-Unis, Walter Stanborough Sutton ${ }^{2}$, en observant la spermatogenèse chez une sauterelle, note l'analogie entre la séparation des chromosomes homologues et la disjonction des gènes; il en conclut que les chromosomes devraient représenter le support matériel de l'hérédité [2]. À partir de cette théorie de Sutton-Boveri, tout est allé très vite. Ces unités individuelles indépendantes, matérialisant ainsi les «particules élémentaires» de Mendel, Thomas Morgan (1866-1945) et son équipe vont les rendre tangibles en étudiant les chromosomes de Drosophila melanogaster. Ayant choisi ce diptère comme matériel expérimental, Morgan n'accepte que les faits: tout doit être étayé par des données scientifiques reproductibles. La rigueur extrême des observations des drosophilistes et de leurs impressionnantes statistiques (81070 comptages en 1910, première année de travail de la fly squad) aboutit à une cartographie complète du génome de $D$. melanogaster et fut couronnée en 1933 par le prix Nobel. Cette exigence était indispensable car initialement de redécouvrir et dont Theodor Boveri (1862-1915) s'inspirait pour interpréter ses observations: chaque espèce animale possèderait un nombre constant de chromosomes, et ceux-ci seraient le support de l'hé-

${ }^{1}$ Ils provenaient de trois «aliénés », traités par castration en raison de leur comportement: self-abuse coupled with certain phases of insanity, à l'asile psychiatrique du Texas. On le voit, se masturber sans modération était périlleux à l'époque.
Morgan était hostile aux thèses de Mendel. Il lui fallait un faisceau de preuves irréfutables pour admettre la théorie chromosomique de l'hérédité et le rôle des chromosomes $X$ et $Y$ dans la détermination sexuelle. 
Car, dès 1905, ce rôle avait été clairement démontré. Mais qui connaît la personne ayant fait cette découverte fondamentale? Qui parmi les lecteurs de Médecine/Sciences peut citer son nom?

Elle n'a cependant pas été totalement oubliée. Dans la littérature anglo-saxonne, certains expliquent pourquoi ces notions de base sur I'X et l'y, qui sont le résultat exclusif de son travail, sont volontiers attribuées à Thomas Morgan, ou à Edmund Wilson (1856-1939) [3]. Ils invoquent «l'effet Matthieu » [4]: on ne prête qu'aux riches, dans le monde scientifique comme ailleurs...

En France, en revanche, on ne la trouve mentionnée dans aucun manuel de génétique. Elle est restée inconnue de la plupart des scientifiques et seul le livre d'Olivier Postel-Vinay, paru en 2007 et destiné au grand public, contient quelques pages sur sa vie et sa découverte [5]. Il était donc temps de réparer cette omission et de faire connaître Nettie Mary Stevens aux lecteurs de Médecine/Sciences.

\section{Une vocation tardive}

Nettie Mary Stevens est la troisième enfant d'une famille aisée de la nouvelle Angleterre. Son père, Ephraïm, construit des maisons à charpente de bois et sa prospérité s'accroîtra encore après la guerre de sécession qui sévit au moment de la naissance de Nettie en 1861. Une sœur, Emma, naît en 1863. Mais leur mère, Julia, meurt peu de temps après, ainsi que les deux frères aînés. Nettie et $\varepsilon m m a$ seront donc élevées par leur belle mère, Ellen Thompson, avec laquelle leur père s'est remarié en 1865 . Les deux sœurs font de bonnes études, vont à la Westfield Normal School (Massachusetts), une école normale qui forme des enseignants et dont les statuts précisent qu'elle est ouverte à tous sans distinction de nationalité, d'âge ou de sexe. Elle devient donc institutrice en 1880 et enseigne dans cette même école pendant plusieurs années. Elle travaille ensuite à la bibliothèque municipale de Westford, ville où elle habite ainsi que sa famille. Comme beaucoup de femmes célibataires, elle veut à la fois être indépendante financièrement, mais aussi épargner un peu d'argent. Car elle a un projet. En 1895, elle décide de quitter la côte Est et part seule en Californie pour s'inscrire à l'Université de Leland Stanford. Créée quelques années auparavant, cette Université - fait assez rare - accepte les filles. Ses parents la rejoindront un an plus tard.

Stanford est déjà une université prestigieuse, dotée d'enseignants renommés et ayant établi des contacts avec de nombreux chercheurs. Les échanges sont particulièrement fructueux avec l'Institut zoologique de Würzburg où Boveri a commencé ses travaux sur la divi- sion cellulaire, la méiose, la fécondation ${ }^{3}$. La Stazione Zoologica, célèbre pour son Centre de Biologie Marine à Naples est aussi très visitée. Créée deux décennies plus tôt par Anton Dohrn, un Allemand qui avait su la mettre en relation avec un réseau de zoologistes de nombreux pays, elle laissait à ceux qui y séjournaient un souvenir enchanté. Le biologiste Edmund Beecher Wilson, dont nous aurons l'occasion de reparler, s'y était rendu en 1882 et écrit à propos de son stage à la station: «It was a rich combinaison of serious effort, new friendships, incomparable beauty of scenery [...] new vistas of scientific work opening before me; in short, a realization of my wildest, most unreal, dreams».

C'est donc dans cette atmosphère éclairée et ouverte sur le monde qu'à 35 ans Nettie entame son cursus de biologie. Elle travaille sur les infusoires ciliés ${ }^{4}$ [6]. Elle obtient son Bachelor of Arts en 1899 et son Master of Arts en 1900, et elle poursuit ses études sous la direction de Franck MacFarlane, devenu professeur après avoir passé plusieurs années en Europe. Elle se forme à l'histologie et chaque été, travaille au Hopkins seaside laboratory à Pacific Grove, seconde station de biologie marine des États-Unis.

\section{Un parcours cohérent}

Nettie décide alors de retourner sur la côte Est pour s'inscrire au Bryn Mawr College. Cette école de Pennsylvanie, fondée par des Quakers une quinzaine d'années auparavant, est réservée aux filles. Contrairement aux pays européens ${ }^{5}$, les États-Unis avaient créé un certain nombre d'écoles supérieures pour femmes. Mais le niveau des études n'était pas toujours équivalent à celui des écoles réservées aux garçons. Pourtant, en l'occurrence, Nettie avait fait un bon choix car l'enseignement y était excellent, surtout en biologie cellulaire. Après $\varepsilon d m u n d ~ B$. Wilson qui y avait enseigné de 1885 à 1891, Thomas Morgan lui avait succédé. Il est professeur au moment où Nettie s'y inscrit.

En 1901, elle obtient une bourse qui lui permet de se rendre en Europe où elle réalise le rêve de nombreux étudiants de l'époque: travailler à la station zoologique de Naples, et à Würztburg chez le professeur Boveri. Il y accueillait d'autant plus volontiers des étudiants des États-Unis que les échanges se faisaient en anglais car il avait épousé une biologiste américaine, Marcella 0'Grady. Formée, elle aussi à Bryn Mawr School et venue pour un stage, elle y était restée et participait aux travaux de son mari6.

Sans doute Nettie garde de son séjour à Würtsburg un bon souvenir puisqu'elle s'efforce de décrocher une nouvelle bourse pour y retourner en 1908. Mais contrairement à d'autres (comme Edna Carter qui

\footnotetext{
${ }^{3}$ Ultérieurement sa théorie sur les mécanismes pathogéniques des cancers lui valut une renommée plus grande encore.

${ }^{4}$ Protozoaires unicellulaires pourvus de cils et vivant en milieu aquatique (I'un d'eux porte le nom de Boveri : ciliate infusoria boveria).

${ }^{5}$ Une institution comme le New York Medical College for Women n'avait pas d'équivalent en Europe. Vers 1900 , les filles désirant faire des études supérieures étaient très rares : à Paris, par exemple, sur 615 étudiants reçus docteurs en médecine, il n'y avait que 11 femmes - et parmi elles une seule Française - les autres venant pour la plupart de l'Europe de l'Est.

${ }^{6}$ Marcella collabora avec son mari jusqu'à sa mort (1915), avant de retourner aux États-Unis où elle obtint un poste au département des sciences du College Albertus Magnus, nouvellement créé pour les femmes à New Haven. Une récente étude souligne son rôle - totalement inédit - dans l'œuvre de T. Boveri [7]. 
devint une amie de Marcella Boveri et y reçut un PhD pour ses travaux sur les rayons $X$ ), Nettie ne semble pas - pas plus du reste que Thomas Morgan -, avoir laissé de son passage une impression favorable à Boveri ${ }^{7}$.

Sur ses voyages et les autres aspects de sa vie, ses lettres n'apportent que peu de renseignements. À 40 ans, toujours célibataire, elle se passionne surtout pour ses recherches. Mais, étant peu rémunérée, et ayant parfois des difficultés à obtenir des subsides, il lui arrive d'hésiter entre un poste mieux payé et la poursuite de son travail. Dans ses démarches pour obtenir des fonds auprès de la Carnegie Intitution de Washington, elle écrit une phrase que, malheureusement, bien des chercheurs d'aujourd'hui pourraient reprendre à leur compte: "I heard rumor... for research work in which the members were to receive salaries and give their time to investigation. That is exactly I should like, an opportunity to devote my time to research work and freedom from anxiety over the money question ». En réponse le 27 juin 1903, on lui fait savoir que les crédits sont épuisés. Elle renouvelle sa demande en novembre de la même année. Elle bénéficie d'un appui chaleureux du président de Bryn Mawr, et de son professeur, Thomas Morgan, plus jeune qu'elle de quelques années, mais qui avait commencé sa carrière beaucoup plus tôt. II écrit: «Miss Stevens has not only the training but she has the natural talent that I believe much rarer to find. She has an independant and original mind and does thorougly whatever she undertakes... ». Wilson, à l'époque à I'Université de Columbia, continue à suivre attentivement les recherches du Bryn Mawr College. II appuie lui aussi la demande de Nettie Stevens et écrit: «I know Miss Stevens'work very well and it is a very independant and admirable character from every point of view... ». Mais en février 1904, elle n'a toujours pas de nouvelles de sa demande. Une réponse positive est essentielle pour qu'elle puisse continuer, car son salaire de Research Fellow doit s'arrêter en mars. Elle écrit: «It is very important that I should know at once wether the Research Assistants have been appointed and what the fate of my application has been ». Ce n'est que le 26 mars que Morgan est prévenu que les crédits sont accordés à son élève. Le 28 mars, elle reçoit enfin une notification officielle. Il lui est demandé d'envoyer à l'institution un rapport sur les travaux en cours, ainsi qu'un résumé du rapport final.

${ }^{7}$ Dans une lettre à l'embryologiste Hans Spemann, il écrit: «Miss Stevens and the woman who accompanied her have happily gone away again. They are pure bloodsuckers; each one has gone off from here with one or two completed works, just like Morgan ».

\section{Le rôle des chromosomes sexuels : état des lieux en 1900}

La redécouverte quasi simultanée des travaux de Mendel par les botanistes Hugo de Vries, Carl Correns et Erich Tchermack von Seysenegg, survenue juste en cette première année du $X X^{e}$ siècle fut déterminante pour la compréhension des mécanismes de reproduction. Mais elle n'a pas ébranlé immédiatement les conceptions qui régnaient alors sur l'embryologie, le développement, et plus précisément l'orientation sexuelle des embryons. Ces données se divisent en trois catégories bien décrites dans l'article de Maienschein [8]:

1. Les tenants des facteurs externes: le sexe des individus dépend de facteurs environnementaux qui interviendraient sur l'œuf ou au cours du développement de celui-ci.

2. Parmi les partisans des facteurs internes qui conditionneraient l'orientation sexuelle des embryons, les «internalistes » se divisent en deux groupes:

- Des embryologistes qui, à la suite de Wilhelm Roux, se basent sur l'observation expérimentale du développement des embryons et accordent une place prépondérante au cytoplasme (théorie que soutenait initialement T.H. Morgan) ; ils rejettent l'hypothèse d'une action d'éléments morphologiques intracellulaires.

- Des histologistes examinant au microscope le processus de spermatogenèse et de la fécondation. Boveri en est le chef de file avec ses travaux sur l'ascaris et les oeufs d'oursin ${ }^{8}$. En 1902, Sutton décrit l'étape réductionnelle de la spermatogénèse chez la sauterelle Brachystola magna: appariement des 11 paires plus un chromosome non apparié et à l'étape suivante, réduction en 11 chromosomes uniques, plus le chromosome non apparié [9].

La même année, son professeur, un zoologiste de l'université du Kansas, Clarence Erwin McClung récapitule les observations faites sur cet élément qui ne s'apparie pas et qui fut décrit précédemment comme «nucléole chromatinien » ou «petit chromosome», puis chromosome «accessoire» [10]. Il suggère que celui-ci peut jouer un rôle dans la

${ }^{8}$ Après une fécondation normale, les cellules ont 36 chromosomes et les 4 premières cellules, séparées, peuvent donner chacune des embryon viables. Si l'ovule est vidé de son matériel et fécondé par deux spermatozoïdes, on trouve (entre autres) des cellules à 36 chromosomes, mais elles ne sont pas viables.

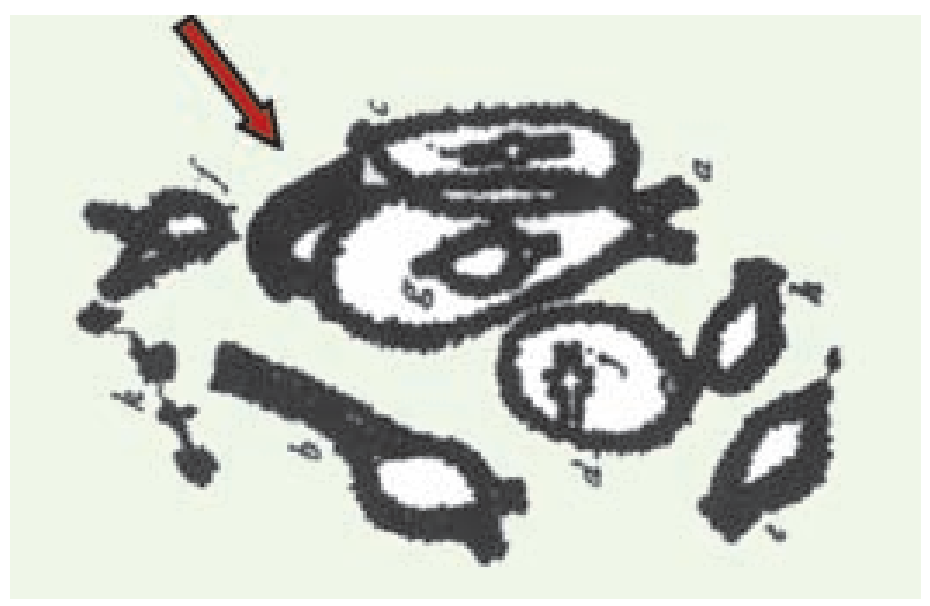

Étape réductionnelle de la spermatogenèse avec le chromosome accessoire (flèche). 
détermination sexuelle. Chez la sauterelle Xiphidium fasciatum, ce serait l'environnement maternel qui interviendrait dans le choix du spermatozoïde, et seuls les œufs porteurs du chromosome accessoire deviendraient des mâles. Ces déductions, toutes erronées puisqu'il s'agissait en fait du chromosome $X$ et que l'environnement maternel n'a pas la capacité de choisir, donnaient néanmoins à réfléchir.

3. Une détermination intrinsèque ou «mendélienne» de la sexualité, conditionnée par des facteurs héréditaires, les recherches étant basées sur l'observation des divisions cellulaires et des études statistiques sur la descendance (sexe ou modifications phénotypiques).

\section{Des publications rapprochées}

Débarrassée de ses soucis d'argent, Nettie Stevens a donc désormais la liberté de poursuivre ses objectifs. Elle les a définis clairement: elle veut étudier «les aspects histologiques des problèmes de l'hérédité en relation avec les lois de Mendel ». Elle connaît les travaux de McClung. En observant les cellules germinales des pucerons, elle n'a pas retrouvé le chromosome accessoire qu'il a décrit, et si elle est convaincue que le sexe est déterminé dans l'œuf, elle est persuadée qu'aucune réponse valable n'a été apportée jusqu'à présent.

Bien que Morgan et Wilson aient chaudement recommandé Miss Stevens, ils restent encore sous l'influence des théories environnementales. Ils avaient tous deux publié sur ce sujet $[11,12]$. Le livre de Wilson dans lequel il était écrit «sex as such is not inherited 》 faisait autorité. Ne cautionnant pas son projet, ils laissent

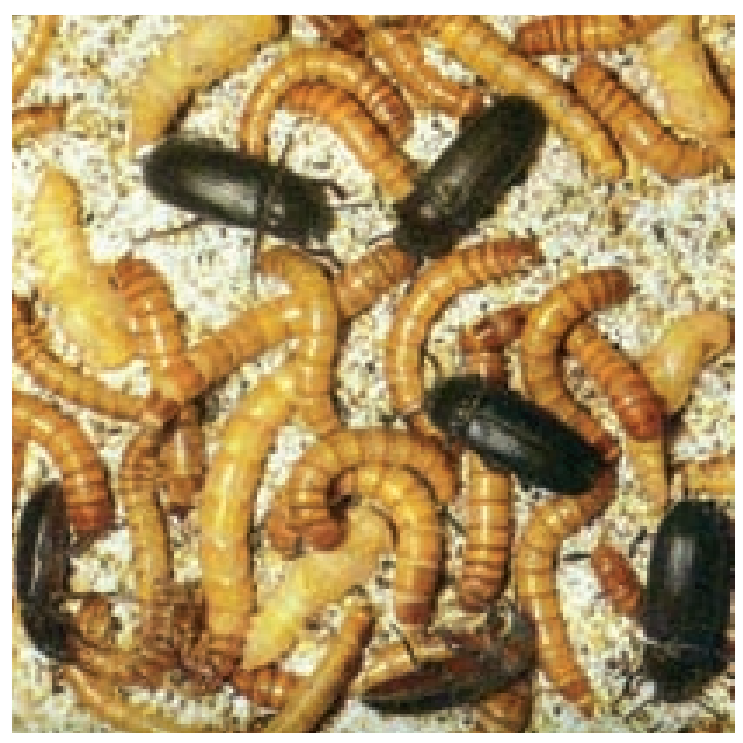

Différents stades du cycle du Ténébrion meunier. donc Nettie faire seule son travail. C'est une chance pour elle : elle devra rendre ses résultats sous son nom, et non pas comme le fruit d'une étude collective dirigée par Morgan.

Le 23 mai, elle a terminé sa monographie et envoie le manuscrit intitulé «Studies in spermatogenesis» pour publication dans la série de monographies de l'Institution Carnégie. Il est aussitôt adressé à Wilson qui fait partie du conseil scientifique et qui répond brièvement le 13 juin: «It is in every way a most admirable piece of work wich is worthly to publication...».

La monographie sort en septembre 1905 [13]. Dans un style précis, Nettie Stevens décrit ses expériences sur la spermatogenèse de larves de Tenebrio molitor (coléoptère vivant dans des denrées sèches, notamment dans la farine, d'où son nom de Ténébrion meunier).

"Puisque les cellules somatiques des larves femelles contiennent 20 grands chromosomes alors que le mâle en contient 19 plus un petit, il semble clair que la détermination sexuelle se fait, non pas par un chromosome "accessoire», mais par cette différence observée dans la paire chromosomique des spermatocytes de premier ordre: les spermatozoïdes qui contiennent le petit chromosome déterminent le sexe mâle et ceux qui contiennent le grand chromosome déterminent le sexe femelle ».

Elle conclut que ce phénomène doit se retrouver dans de nombreux cas, sans exclure l'éventualité de variations dans la forme et la taille des chromosomes d'une espèce à l'autre.

On se saurait être plus clair.

Mais un mois auparavant, Wilson avait publié deux articles: dans le Journal of Experimental Zoology, il montre qu'Anasa tristis (une punaise parasite des citrouilles et autres cucurbitacées) possède dans ses spermatogonies un nombre chromosomique de 21 et non pas de 22. II signale en note en bas de page qu'il a eu connaissance du travail de Miss Stevens [14]. Puis en octobre, dans Science, il admet une relation entre les chromosomes et la détermination du sexe, mais conserve cependant une interprétation semi-environnementale [15].

\section{Épilogue}

En se replaçant dans le contexte de l'époque, il semble naturel que Wilson n'ait pas été immédiatement convaincu des conclusions de Stevens, en raison des variations observées selon les espèces d'animaux étudiés. De même, il n'est pas surprenant que l'article de Wilson ait précédé d'un mois celui de Stevens, ne serait ce que parce qu'il faisait partie du comité éditorial du journal.

Pendant les 8 années qui lui restent à vivre, Nettie continuera l'analyse des hétérochromosomes d'une cinquantaine d'insectes. Elle étudie aussi la reproduction parthénogénétique de diverses espèces de pucerons, parasites de fleurs (aphis rosae, aphis oenotherae), d'insectes, néoptères comme le perce-oreille (forficula), diptères, moustiques et mouches ( 9 espèces avec XY), dont Drosophila melanogaster, à laquelle s'attacheront quelques années plus tard Morgan et son équipe avec le succès que l'on sait. 
Ses publications cessent en 1912, quand elle décède d'un cancer du sein au John Hopkins Hospital de Baltimore à l'âge de 51 ans, sans avoir pu bénéficier du poste de research professorship qui venait d'être créé pour elle à Byrn Mawr.

Si le nom de Nettie Stevens est tombé dans l'oubli pendant des décennies, la faute n'est pas imputable aux chercheurs de son temps. Thomas Morgan a très tôt précisé la part qui revenait à $N$. Stevens et à $\varepsilon$. Wilson dans la découverte de la détermination sexuelle. Certes, à la mort de ce dernier, à l'âge de 83 ans, il consacrera une biographie complète à son aîné de 10 ans qui laissait une œuvre considérable [16]. Mais dès 1912, après le décès de Nettie Stevens, il n'avait pas manqué de lui dédier quelques pages, soulignant l'importance de sa découverte, le scepticisme qui l'accueillit [17], et surtout les qualités de persévérance qu'il fallut à cette scientifique à la carrière si courte pour parvenir à son remarquable accomplissement ${ }^{9}$.

En effet, dans les conditions où se trouvaient les femmes en ce début du XXe siècle, la vie de Nettie Stevens est exemplaire et mérite bien que son nom soit exhumé de l'oubli. $\diamond$

\section{SUMMARY}

\section{Nettie Maria Stevens (1861-1912)}

Nettie M. Stevens is one of the first women who contributed to genetic research. She discovered the role of sex chromosomes in sex determination during the first decade of the twentieth century. However, her discovery has rarely been recognized, and her scientific reputation was eclipsed by that of T.H. Morgan, who was credited for the establishment of modern genetics. Her name is absent from most biology and genetic texts. It is now time to recognize her valuable contribution with this short biography. $\diamond$

\section{RÉFÉRENCES}

1. Painter TS. Studies in mammalian spermatogenesis. II. The spermatogenesis of man. J Exp Zool 1923; 37 : 291-321.

2. Sutton WS. The chromosomes in heredity. Biol Bull Mar Lab 1903; $32: 231-48$.

3. Brush SG. Nettie M. Stevens and the discovery of sex determination by chromosomes. Isis $1978 ; 69: 163-72$.

4. Merton RK. The Matthew effect in science. Science 1968 ; 159: 56-63.

5. Postel-Vinay 0. La revanche du chromosome X. Paris : Lattès, 2007 : $440 \mathrm{p}$.

${ }^{9}$ On trouvera toutes les publications de Nettie Stevens dans un article très complet et publié en 1981 par Marylin B. Ogilvie et Clifford J. Choquette [18].
6. Stevens NM. Studies on ciliate infusora. Proc Calif Acad Sci Zool 1901; 3 (ser 3) : 1-42.

7. Satzinger $\mathrm{H}$. Theodor and Marcella Boveri : chromosomes and cytoplasm in heredity and development. Nat Rev Genet $2008 ; 9: 231-8$.

8. Maienschein J. What determines sex? A study of converging approaches, 1880-1916. Isis 1984 ; $75: 457-80$.

9. Sutton W. On the morphology of the chromosome group in brachystola magna. Biol Bull 1902 ; $4: 24-39$.

10. McClung CE. Notes on the accessory chromosome. Anatomischer Anzeiger 1901;20:220-6.

11. Morgan TH. Recent theories in regard of the determination of sex. Popular Science Monthly $1903 ; 64: 97-116$.

12. Wilson $\varepsilon B$. The cell in development and inheritance, $2^{\text {nd }}$ ed. New York: MacMillan, 1900.

13. Stevens NM. Studies in spermatogenesis with especial referene to the "accessory chromosome». Washington DC: Carnegie Institution Publication, 1906: $\mathrm{n}^{\circ} 36$, part 2.

14. Wilson $E B$. Studies on chromosomes. I. The behaviourof the idiochromosomes in hemiptera. $J$ Exp Zool $2005 ; 2$ : 371-405.

15. Wilson $\varepsilon B$. The chromosome in relation to the determination of sex insects. Science 1905 ; $22: 500-2$.

16. Morgan TH. Edmund Beecher Wilson. Biographical memoirs. Series by the National Academy of Sciences $1940 ; 21: 315-42$.

17. Morgan TH. The sientific work of Miss N.M. Stevens. Science $1912 ; 36: 468-70$.

18. Ogilvie MB, Choquette CJ. Nettie Maria Stevens (1861-1912) : her life and contributions to cytogenetics. Proc Am Philos Soc 1981 ; 125 : 292-311.

\section{TIRÉS À PART}

S. Gilgenkrantz
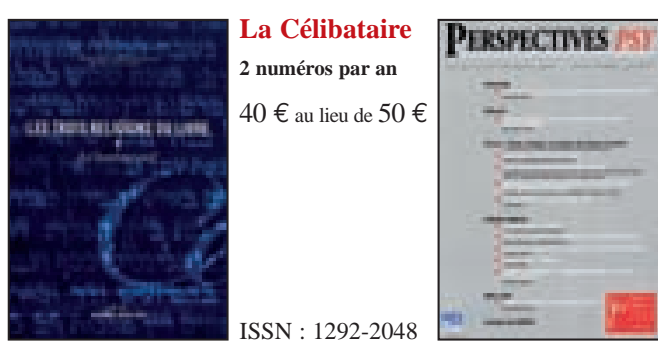

Perspectives Psy 4 numéros par an : $78 €$ au lieu de $100 €$

La Revue la Célibataire s'adresse à tous ceux qui veulent réfléchir aux problèmes de notre société : sexuels, politiques, sociaux, religieux, juridiques, économiques, etc. Elle est une référence pour rendre compte des désarrois actuels.

ISSN : 0031-6032

\section{La Revue Perspective Psychiatriques} est la tribune des discussions et des réflexions multidisciplinaires sur les méthodes nouvelles de compréhension et de traitement des troubles mentaux.

\section{Bon de commande}

NOM Prénom :

\section{Adresse :}

Code postal :

Ville :

E-mail :

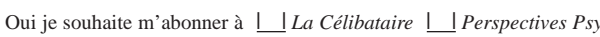

$\square$ Par chèque bancaire ci-joint.

$\square$ Par carte bancaire :

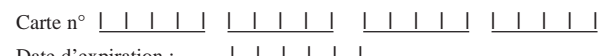

Date d'expiration: $\quad|\quad| \quad|\quad| \quad \mid$

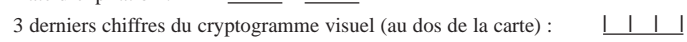

Fait à :

$\mathrm{L}$

Signature : 